\title{
NUMERICAL ANALYSIS OF A CIRCULAR ARCH BUCKLING UNDER THE SYMMETRIC PRESSURE
}

\author{
JAEGWI GO, YOUNGMI CHOI, AND ZHONG BO FANG
}

Received 3 March 2005; Accepted 12 October 2005

The many interesting phenomena, such as snap-through, nonuniqueness, and stability, of a circular arch subjected to the symmetric pressure are studied. The balance of forces of an elemental length leads to a governing equation which is used to investigate stable states of the arch. With specific opening angle $2 a=\pi / 3$, the sensitivities of angle and curvature at a base are surveyed for various of spring constants. The variations of angle and curvature at an edge are almost the same if the spring constant $\tau \geq 10$.

Copyright (c) 2006 Jaegwi Go et al. This is an open access article distributed under the Creative Commons Attribution License, which permits unrestricted use, distribution, and reproduction in any medium, provided the original work is properly cited.

\section{Introduction}

The behavior of an arch under the action of pressure is dependent on the flexural rigidity of the arch, opening angle, and base conditions. Schreyer and Masur [4] accomplished an exact analysis and obtained analytical solutions for shallow arches with clamped bases. A series method was used to investigate the numerical solutions of the buckling shallow arch with clamped bases by Dickie and Broughton [1], while Pi et al. [3] analyzed the in-plane buckling of circular pin-ended and fixed arches with an arbitrary cross-section under uniformly distributed normal pressure around the arch. On the other hand, Go [2] realized many interesting phenomena, such as snap-through, nonuniqueness, and stability, by considering a circular arch, which is restrained with torsional spring at the bases, under normal uniform loading.

Elastic, inextensible, and thin circular arches subjected to the action of symmetric pressure, for example, gravity, are considered. The bases of the arches are restrained with torsional spring (Figure 1.1). Our main concerns are to analyze the susceptibility of the arch for various spring constants and stability of each bifurcation curve. For our purpose, the pressure-base angle and pressure-base curvature plots for the variations of angle and curvature and the pressure-base area for stability of each bifurcation curve are used.

In Section 2, the method of equilibrium of elemental segment is used to formulate the governing equation. The balance of normal and shear stresses of the elemental segment 
2 Numerical analysis of a buckling arch

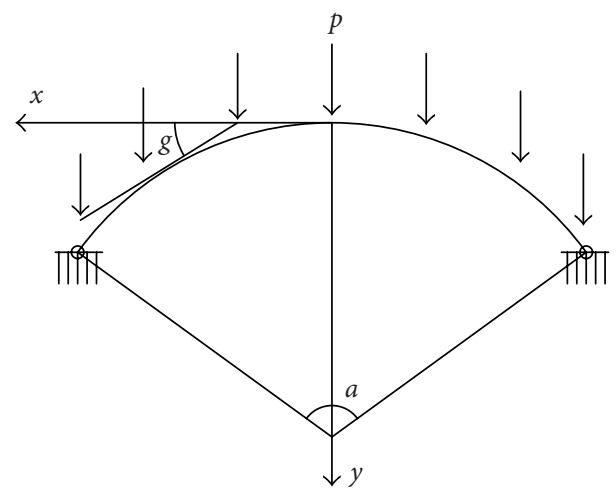

Figure 1.1. Symmetric load uniformly distributed around arch.

(Figure 2.1) leads to a nonlinear ordinary boundary value problem. Based on the fourthorder Runge-Kutta method, we use the shooting method to solve the boundary value problem in Section 3.

\section{Derivation of equation}

The balance of forces in the normal and tangential directions and the balance of local moment are used to derive the governing equation. According to the bending theory, it leads to

$$
\begin{gathered}
T d g-d S-q_{n}^{\prime} d s^{\prime}=0, \\
d T+S d g+q_{t}^{\prime} d s^{\prime}=0, \\
d M-S d s^{\prime}=0,
\end{gathered}
$$

where $T, S, q_{n}^{\prime}$, and $q_{t}^{\prime}$ are tension, shear, normal, and tangential stresses on the surface, respectively. The $g$ represents the local angle of inclination (Figure 2.2) and $s^{\prime}$ the arc length. Moreover, we assume that the moment is proportional to local curvature, that is,

$$
M=\mathrm{EI} \frac{d g}{d s^{\prime}},
$$

where EI is the flexural rigidity. From (2.3) and (2.4),

$$
S=\mathrm{EI} \frac{d^{2} g}{d s^{\prime 2}},
$$

and from (2.1) and (2.2),

$$
-S \frac{d g}{d s^{\prime}}-q_{t}^{\prime}=\frac{d^{2} S}{d s^{\prime 2}} \frac{d s^{\prime}}{d g}-\frac{d S}{d s^{\prime}} \frac{d^{2} g}{d s^{\prime 2}}\left(\frac{d s^{\prime}}{d g}\right)^{2}-q_{n}^{\prime} \frac{d^{2} g}{d s^{\prime 2}}\left(\frac{d s^{\prime}}{d g}\right)^{2} .
$$

The combination of (2.5) and (2.6) provides

$$
\mathrm{EI} \frac{d^{4} g}{d s^{\prime 4}} \frac{d g}{d s^{\prime}}-\mathrm{EI} \frac{d^{3} g}{d s^{\prime 3}} \frac{d^{2} g}{d s^{\prime 2}}-q_{n}^{\prime} \frac{d^{2} g}{d s^{\prime 2}}+\mathrm{EI} \frac{d^{2} g}{d s^{\prime 2}}\left(\frac{d g}{d s^{\prime}}\right)^{3}+q_{t}^{\prime}\left(\frac{d g}{d s^{\prime}}\right)^{2}=0
$$




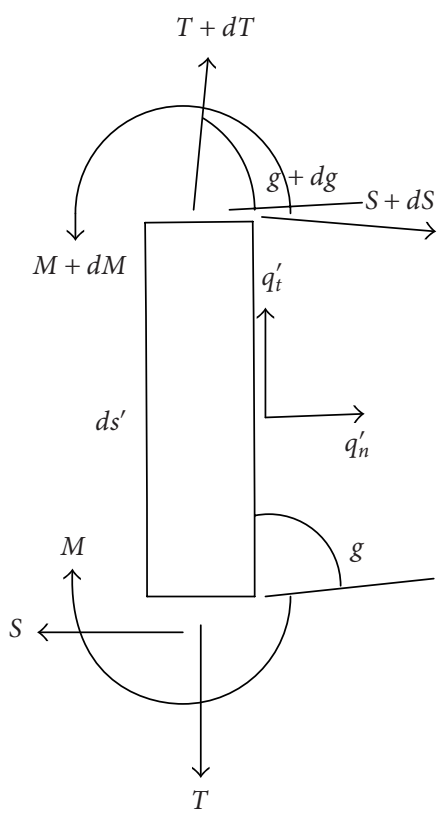

Figure 2.1. An elemental length.

and through normalizing arc length and stress by $s=s^{\prime} / R$ and $q_{n}=q_{n}^{\prime} R^{3} /$ EI, it is obtained the nonlinear equation

$$
g_{s s s} g_{s}-g_{s s s} g_{s s}-q_{n} g_{s s}+g_{s s} g_{s}^{3}+q_{t} g_{s}^{2}=0 .
$$

Note that, under the vertical pressure, the normalized normal and tangential stresses are written by $q_{n}=-p \cos g$ and $q_{t}=p \sin g$ (Figure 2.2), and the $(x, y)$-cartesian coordinates normalized by $R$ are related to $g$ by

$$
x_{s}=\cos g(s), \quad y_{s}=\sin g(s) .
$$

Since the total angle change is resisted by an additional moment at the bases with torsional spring constants, the boundary conditions are

$$
\begin{gathered}
\tau^{\prime}(g(-a)+a)-\operatorname{EI}\left(g_{s^{\prime}}(-a)-\frac{1}{R}\right)=0, \\
\tau^{\prime}(g(a)-a)+\operatorname{EI}\left(g_{s^{\prime}}(a)-\frac{1}{R}\right)=0 .
\end{gathered}
$$

The normalized forms then are given by

$$
\begin{aligned}
& (g(0)+a)-\tau\left(g_{s}(0)-2 a\right)=0, \\
& (g(1)-a)+\tau\left(g_{s}(1)-2 a\right)=0,
\end{aligned}
$$




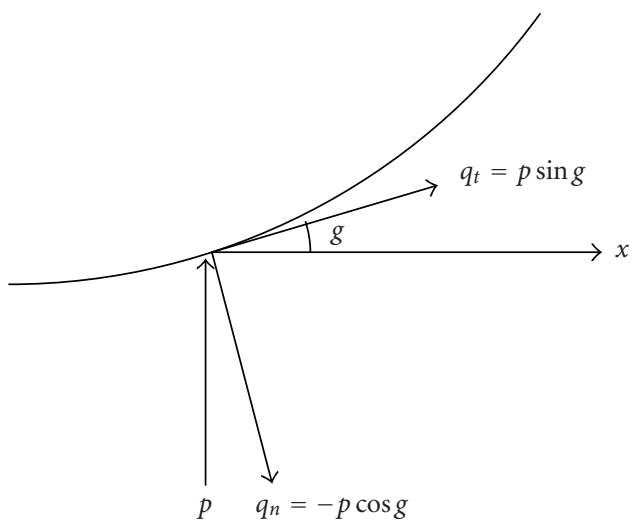

Figure 2.2. The strength of stresses.

where $\tau=\mathrm{EI} / \tau^{\prime} R$. The $\tau=0$ and $\tau=\infty$ imply fixed and hinged base bucklings, respectively.

\section{Numerical results and discussions}

In this section, the equilibrium states of arches are investigated, for each given angle $a$ and pressure $p$, using numerical tools. We transform (2.8) by $g(s)=\tilde{g}(1-s)$ and dropping tilde, and reduce, after dividing by $g_{s}^{2}$ and integrating, to

$$
g_{s s s}+\frac{1}{2} g_{s}^{3}-c g_{s}-q_{t} s g_{s}-q_{n}=0
$$

to remove the risk of singularity. The $c$ is an integral constant. Equation (3.1) is shoot based on the fourth-order Runge-Kutta. The boundary conditions are

$$
\begin{array}{ccc}
(g(0)-a)-\tau\left(g_{s}(0)+2 a\right)=0, & (g(1)+a)+\tau\left(g_{s}(1)+2 a\right)=0, \\
x(0)=0, \quad x(1)=\frac{\sin a}{a}, & y(0)=0, \quad y(1)=0 .
\end{array}
$$

Some methods to reduce the target boundary conditions were investigated in [2].

We pay attention to describe the behaviors of a circular arch of opening angle $a=\pi / 6$ for various spring constants $\tau$. Numerical results are displayed using a curve of pressurebase curvature and pressure-base angle plot for each case. Some representative solutions are depicted to show the process of collapse of the arch through snap-through.

Figure 3.1 presents the variation of a base angle versus the uniform pressure $p$. Two types of configuration, symmetric (branches $C, D$, and $E$ ) and antisymmetric (branches $A$ and $B$ ) modes, are observed. Like the results of Go [2], the antisymmetric bifurcation curve is an ellipse. The deformed symmetric shapes between the branchs $C$ and $E$ are different. Both bases bend downward in branch $E$ (Figure 3.3), while the shape in branch 


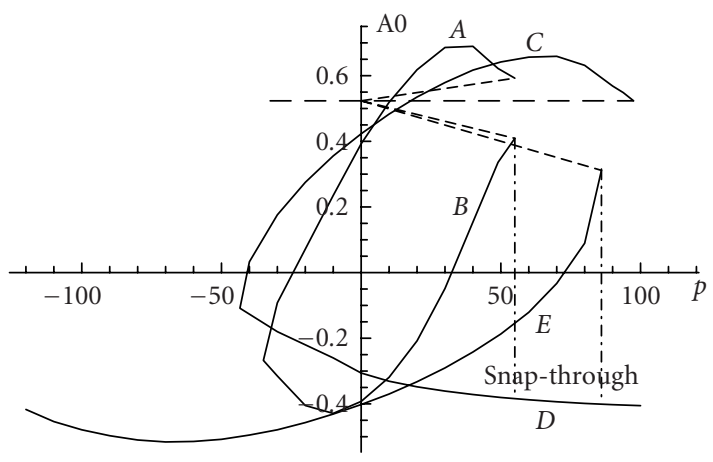

Figure 3.1. $A 0=$ angle at $s=0, \tau(0)=\tau(1)=\infty, a=\pi / 6$.

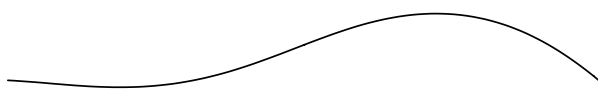

(a)

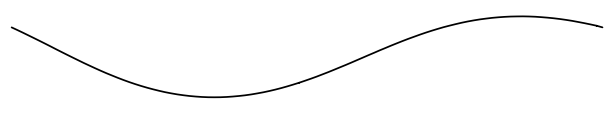

(b)

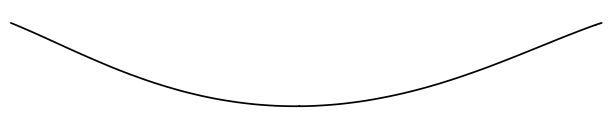

(c)

Figure 3.2. (a) $\tau=\infty, a=\pi / 6, p=30$; (b) $\tau=\infty, a=\pi / 6, p=-10$; (c) $\tau=\infty, a=\pi / 6, p=60$.

$C$ bends downward at mid point. Figures 3.2 and 3.3 show the process of deformation of the arch of each branch $C$ and $E$ as pressure decreases.

Not all equilibrium solutions, however, are stable. The stability of each branch is studied using pressure-base "area." Area is defined by the difference between the trivial circular arch and the deformed arch for each given pressure. Stable means that the area increases as pressure increases, otherwise unstable. Only the branch $D$ is stable and the others (branches $A, B, C$, and $E$ ) are unstable (Figure 3.4).

Figure 3.5 displays the variation of angle at $s=0$ for different spring constants $\tau(0)=$ $0, \tau(0)=1, \tau(0)=10$, and $\tau(0)=100$ with $\tau(1)=0$. The pressures for snap-through mode is $p_{s} \approx 49, p_{s} \approx 54, p_{s} \approx 60$, and $p_{s} \approx 68$ for each spring constant $\tau=\infty, \tau=100$, $\tau=10$, and $\tau=1$, which can be understood as a reasonable modelling. In similar manner, the changes of curvature at $s=1$ are investigated for some kinds of spring constants $\tau(1)=0, \tau(1)=1, \tau(1)=10$, and $\tau(1)=100$ with $\tau(0)=0$ in Figure 3.6. In both cases 
6 Numerical analysis of a buckling arch

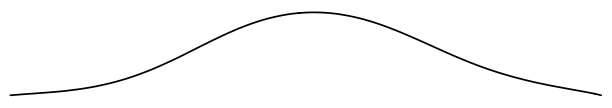

(a)

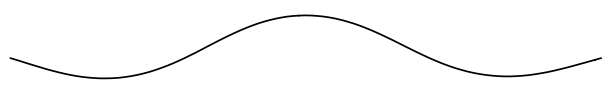

(b)

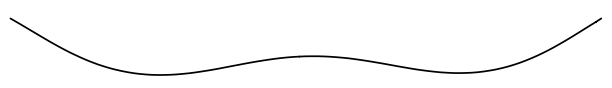

(c)

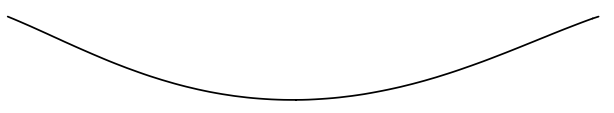

(d)

Figure 3.3. (a) $\tau=\infty, a=\pi / 6, p=80$; (b) $\tau=\infty, a=\pi / 6, p=30$; (c) $\tau=\infty, a=\pi / 6, p=-70$; (d) $\tau=\infty, a=\pi / 6, p=60$.

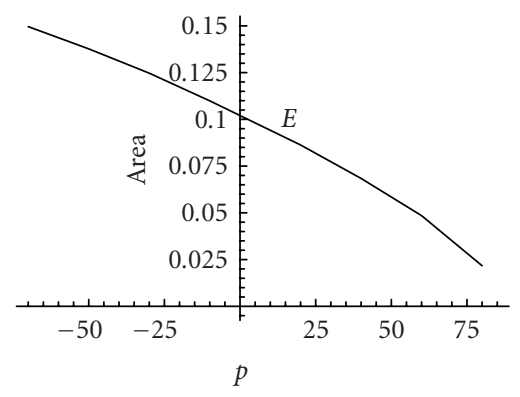

(a)

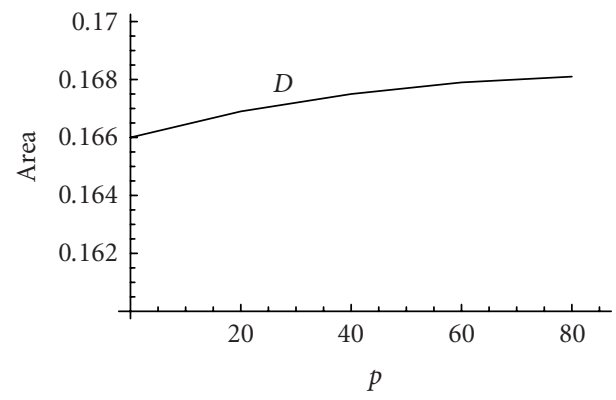

(c)

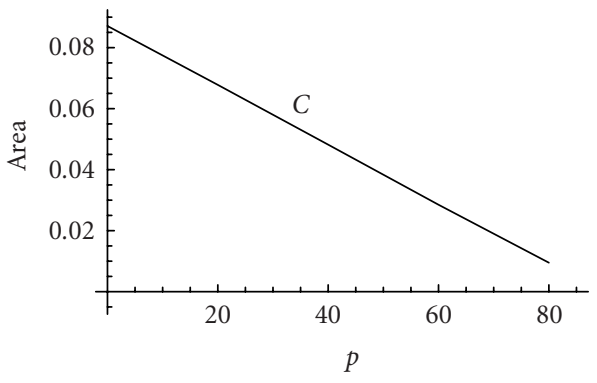

(b)

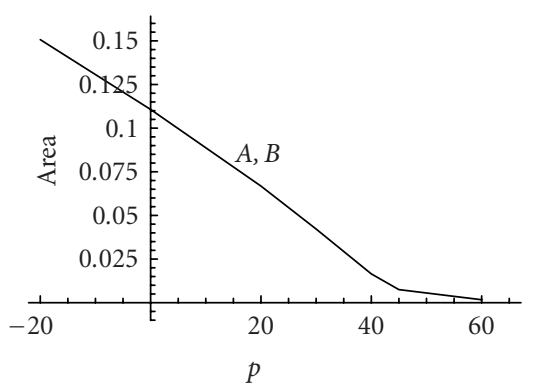

(d)

Figure 3.4. (a) Branch $E$, $\tau=\infty, a=\pi / 6$; (b) branch $C$, $\tau=\infty, a=\pi / 6$; (c) branch $D, \tau=\infty, a=\pi / 6$; (d) branch $A, B, \tau=\infty, a=\pi / 6$. 


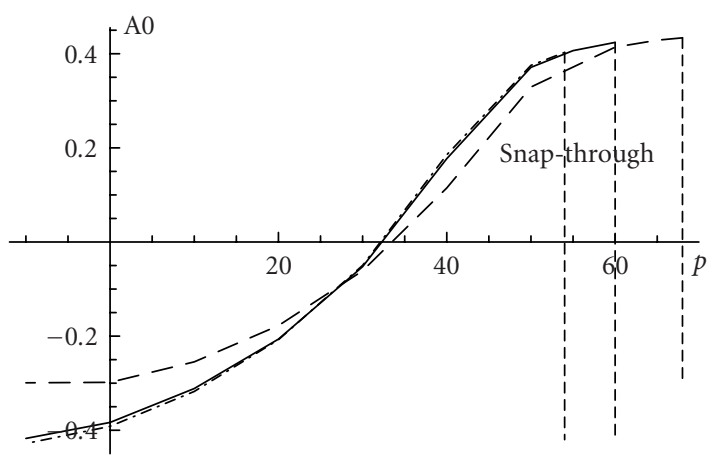

$---\tau(0)=1$

$\tau(0)=10$

... $\quad \tau(0)=100$

Figure 3.5. $A 0=$ angle at $s=0, a=\pi / 6, \tau(1)=0$.

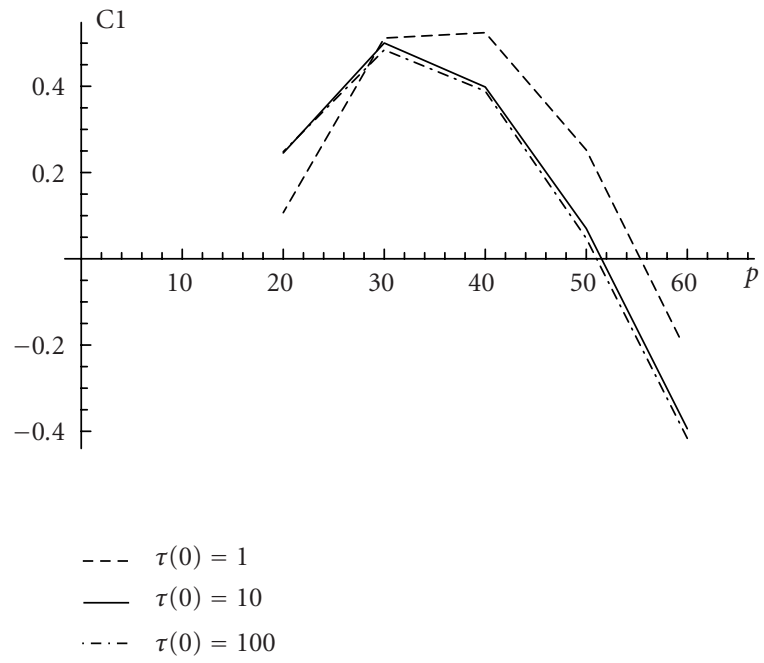

Figure 3.6. $C 1=$ curvature at $s=1, a=\pi / 6, \tau(1)=0$.

(Figures 3.5 and 3.6), the changes of angle or curvature are almost the same if spring constant $\tau \geq 10$.

\section{Appendix}

The program files (using $C^{++}$computer language) are attached. 
8 Numerical analysis of a buckling arch

Table A.1. Initial data.

\begin{tabular}{|c|c|c|c|c|c|}
\hline$\tau(0)=\tau(1)$ & $\alpha$ & $p$ & $\theta(0)$ & $c$ & $\theta_{s s}(0)$ \\
\hline$\infty$ & $\frac{\pi}{6}$ & 100 & -0.4054 & 67.950016 & 20.2458 \\
\hline$\infty$ & $\frac{\pi}{6}$ & 80 & -0.3986 & 50.373826 & 18.263 \\
\hline$\infty$ & $\frac{\pi}{6}$ & 60 & -0.3875 & 32.957667 & 16.047 \\
\hline$\infty$ & $\frac{\pi}{6}$ & 40 & -0.3715 & 15.752357 & 13.481 \\
\hline$\infty$ & $\frac{\pi}{6}$ & 20 & -0.348 & -1.46006 & 10.36 \\
\hline$\infty$ & $\frac{\pi}{6}$ & 0 & -0.306 & -18.7281 & 6.097 \\
\hline$\infty$ & $\frac{\pi}{6}$ & -10 & -0.258 & -27.4485 & 2.68 \\
\hline$\infty$ & $\frac{\pi}{6}$ & -40 & 0.033 & -66.55082 & -20.3 \\
\hline$\infty$ & $\frac{\pi}{6}$ & -20 & 0.275 & -70.85218 & -29.6 \\
\hline$\infty$ & $\frac{\pi}{6}$ & 0 & 0.423 & -73.07093 & -33.1 \\
\hline$\infty$ & $\frac{\pi}{6}$ & 20 & 0.535 & -75.206311 & -34.3 \\
\hline$\infty$ & $\frac{\pi}{6}$ & 40 & 0.617 & -77.7038 & -33.02 \\
\hline$\infty$ & $\frac{\pi}{6}$ & 60 & 0.6565 & -80.97574 & -28.02 \\
\hline$\infty$ & $\frac{\pi}{6}$ & 80 & 0.6315 & -85.75932 & -17.65 \\
\hline$\infty$ & $\frac{\pi}{6}$ & 86 & 0.3115 & -83.2982 & 14.4 \\
\hline$\infty$ & $\frac{\pi}{6}$ & 60 & -0.121 & -87.220119 & 38.3 \\
\hline$\infty$ & $\frac{\pi}{6}$ & 40 & -0.242 & -88.61008 & 40.87 \\
\hline$\infty$ & $\frac{\pi}{6}$ & 20 & -0.3325 & -90.27676 & 41 \\
\hline$\infty$ & $\frac{\pi}{6}$ & 0 & -0.402 & -92.05987 & 39.35 \\
\hline$\infty$ & $\frac{\pi}{6}$ & -20 & -0.457 & -95.015 & 36.8 \\
\hline$\infty$ & $\frac{\pi}{6}$ & -40 & -0.495 & -98.4632 & 32.8 \\
\hline$\infty$ & $\frac{\pi}{6}$ & -60 & -0.5145 & -103.31382 & 27.3 \\
\hline$\infty$ & $\frac{\pi}{6}$ & -80 & -0.51 & -110.40713 & 20.3 \\
\hline
\end{tabular}


Table A.1. Continued.

\begin{tabular}{cccccc}
\hline$\infty$ & $\frac{\pi}{6}$ & -100 & -0.479 & -120.91787 & 11.8 \\
$\infty$ & $\frac{\pi}{6}$ & -120 & -0.417 & -135.0085 & 0.4 \\
$\infty$ & $\frac{\pi}{6}$ & 55 & 0.409 & -53.09718 & 4.2 \\
$\infty$ & $\frac{\pi}{6}$ & 40 & 0.1865 & -40.88411 & 10.76 \\
$\infty$ & $\frac{\pi}{6}$ & 20 & -0.208 & -36.88867 & 17 \\
$\infty$ & $\frac{\pi}{6}$ & 0 & -0.3925 & -36.7712 & 15.45 \\
$\infty$ & $\frac{\pi}{6}$ & -20 & -0.405 & -39.88732 & 7.8 \\
$\infty$ & $\frac{\pi}{6}$ & -35 & -0.2675 & -50.40328 & -2.26 \\
\hline
\end{tabular}

Table A.2. Initial data.

\begin{tabular}{|c|c|c|c|c|c|c|c|}
\hline$\tau(0)$ & $\tau(1)$ & $\alpha$ & $p$ & $\theta(0)$ & $\theta_{s}(0)$ & $c$ & $\theta_{s s}(0)$ \\
\hline 100 & 100 & $\frac{\pi}{6}$ & 50 & 0.37535 & -1.04868 & -48.3 & 5.345652 \\
\hline 100 & 100 & $\frac{\pi}{6}$ & 30 & -0.049655 & -1.05293 & -38.02098 & 15.5 \\
\hline 100 & 100 & $\frac{\pi}{6}$ & 10 & -0.317649 & -1.05561 & -36.580012 & 16.84187 \\
\hline 100 & 100 & $\frac{\pi}{6}$ & -10 & -0.428156 & -1.056715 & -37.693106 & 12.7 \\
\hline 10 & 10 & $\frac{\pi}{6}$ & 60 & 0.42408 & -1.05715 & -57.67569 & 3.84 \\
\hline 10 & 10 & $\frac{\pi}{6}$ & 40 & 0.177079 & -1.08185 & -41.0891 & 11.15 \\
\hline 10 & 10 & $\frac{\pi}{6}$ & 20 & -0.206421 & -1.1202 & -37.23299 & 17.1 \\
\hline 10 & 10 & $\frac{\pi}{6}$ & 0 & -0.38342 & -1.1379 & -37.15 & 15.34905 \\
\hline 10 & 10 & $\frac{\pi}{6}$ & -10 & -0.41742 & -1.1413 & -38.06 & 12.4889 \\
\hline 1 & 1 & $\frac{\pi}{6}$ & 60 & 0.413997 & -0.1568 & -57.63635 & 4.55 \\
\hline 1 & 1 & $\frac{\pi}{6}$ & 40 & 0.114797 & -1.456 & -43.1 & 13.85304 \\
\hline 1 & 1 & $\frac{\pi}{6}$ & 20 & -0.177703 & -1.7485 & -40.0776 & 17.32 \\
\hline 1 & 1 & $\frac{\pi}{6}$ & 0 & -0.298203 & -1.869 & -40.26 & 13.63965 \\
\hline 1 & 1 & $\frac{\pi}{6}$ & -10 & -0.299203 & -1.87 & -41.47057 & 9.3 \\
\hline
\end{tabular}


10 Numerical analysis of a buckling arch

Table A.3. Initial data.

\begin{tabular}{|c|c|c|c|c|c|c|c|c|}
\hline$\tau(0)$ & $\tau(1)$ & $\alpha$ & $p$ & $\theta(0)$ & $\theta_{s}(0)$ & $c$ & $\theta_{s s}(0)$ & $\theta_{s}(1)$ \\
\hline 100 & 0 & $\frac{\pi}{6}$ & 60 & 0.325342 & -1.04918 & -61 & 8.89033 & -0.416293 \\
\hline 100 & 0 & $\frac{\pi}{6}$ & 50 & 0.212343 & -1.05031 & -55.3 & 12.4861 & 0.047348 \\
\hline 100 & 0 & $\frac{\pi}{6}$ & 40 & 0.080343 & -1.05163 & -51.11172 & 15.6 & 0.388698 \\
\hline 100 & 0 & $\frac{\pi}{6}$ & 30 & -0.048356 & -1.052917 & -47.54762 & 17.5 & 0.484369 \\
\hline 100 & 0 & $\frac{\pi}{6}$ & 20 & -0.172655 & -1.05416 & -43.85795 & 18.2 & 0.248203 \\
\hline 10 & 0 & $\frac{\pi}{6}$ & 60 & 0.322574 & -1.0673 & -61 & 9.09989 & -0.39386 \\
\hline 10 & 0 & $\frac{\pi}{6}$ & 50 & 0.208574 & -1.0787 & -55.41751 & 12.7 & 0.070238 \\
\hline 10 & 0 & $\frac{\pi}{6}$ & 40 & 0.078574 & -1.0917 & -51.26867 & 15.72 & 0.398303 \\
\hline 10 & 0 & $\frac{\pi}{6}$ & 30 & -0.050927 & -1.10465 & -47.7352 & 17.7 & 0.500319 \\
\hline 10 & 0 & $\frac{\pi}{6}$ & 20 & -0.172426 & -1.1168 & -44.0552 & 18.29 & 0.245279 \\
\hline 1 & 0 & $\frac{\pi}{6}$ & 59.2 & 0.292796 & -1.278 & -61.126 & 10.78 & -0.181222 \\
\hline 1 & 0 & $\frac{\pi}{6}$ & 50 & 0.182796 & -1.388 & -56.5 & 14.309 & 0.252329 \\
\hline 1 & 0 & $\frac{\pi}{6}$ & 40 & 0.061796 & -1.509 & -52.7 & 16.9988 & 0.524258 \\
\hline 1 & 0 & $\frac{\pi}{6}$ & 30 & -0.048204 & -1.619 & -49.3855 & 18.21 & 0.511835 \\
\hline 1 & 0 & $\frac{\pi}{6}$ & 20 & -0.151204 & -1.722 & -45.6494 & 18.15 & 0.106911 \\
\hline
\end{tabular}

\section{Acknowledgment}

This work was supported by the Research Fund of KOSEF-R14-2003-019-01001.

\section{References}

[1] J. F. Dickie and P. Broughton, Stability criteria for shallow arches, Journal of the Engineering Mechanics Division 97 (1971), no. 3, 951-965.

[2] J. Go, Buckling arch under normal pressure, Ph.D. thesis, Michigan State University, Michigan, 2004.

[3] Y.-L. Pi, M. A. Bradford, and B. Uy, In-plane stability of arches, International Journal of Solids and Structures 39 (2002), no. 1, 105-125. 
[4] H. L. Schreyer and E. F. Masur, Buckling of shallow arches, Journal of Engineering Mechanics 92 (1966), no. 4, 1-19.

Jaegwi Go: Department of Mathematics, Chonnam National University, Gwangju 500-757, South Korea

E-mail addresses: gojaegwi@msu.edu; jggo@chonnam.ac.kr; jaegwigo@hanmail.net

Youngmi Choi: Department of Mathematics, Chonnam National University, Gwangju 500-757, South Korea

E-mail address: ymchoi93@hanmail.net

Zhong Bo Fang: Department of Mathematics, Chonnam National University, Gwangju 500-757, South Korea

E-mail address: fangzb7777@hotmail.com 


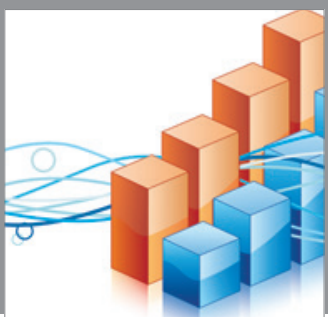

Advances in

Operations Research

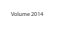

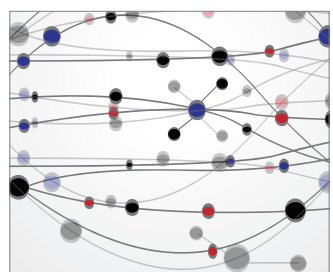

\section{The Scientific} World Journal
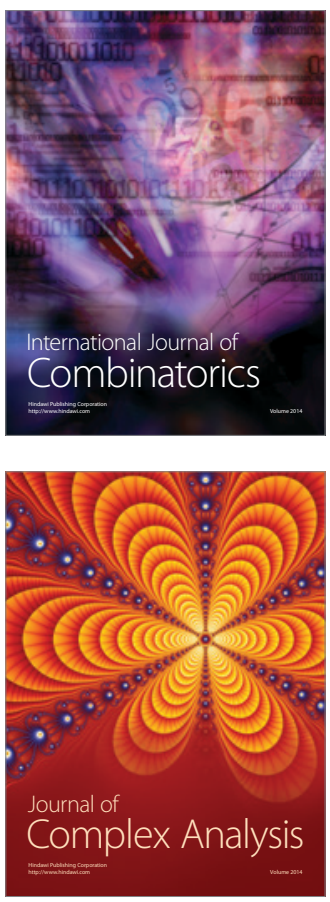

International Journal of

Mathematics and

Mathematical

Sciences
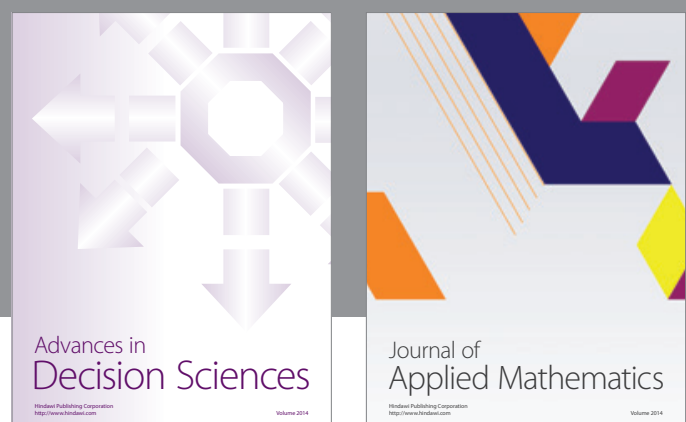

Journal of

Applied Mathematics
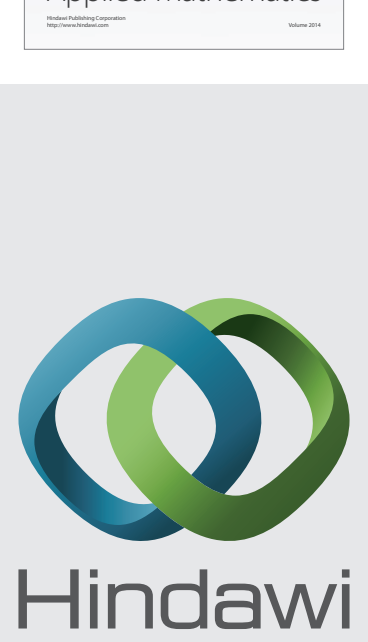

Submit your manuscripts at http://www.hindawi.com
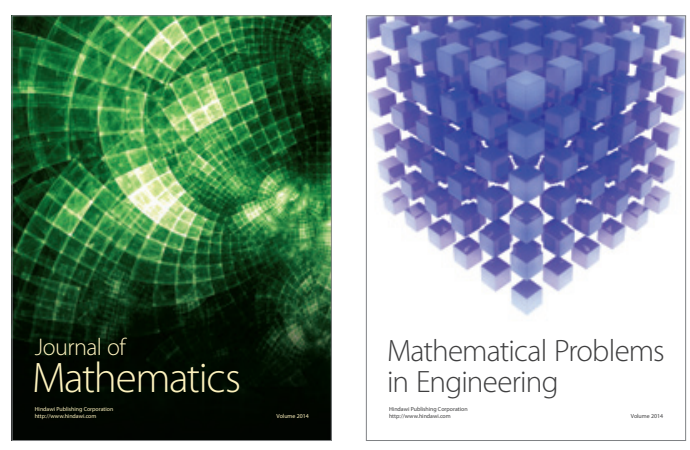

Mathematical Problems in Engineering
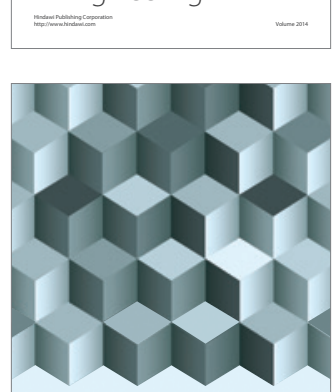

Journal of

Function Spaces
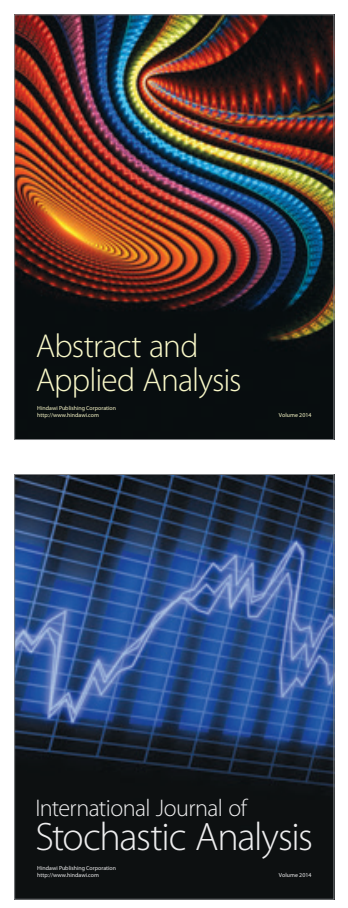

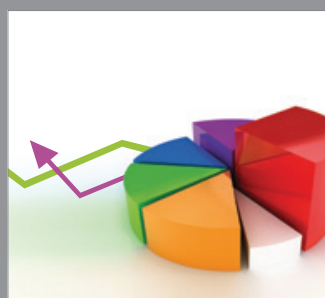

ournal of

Probability and Statistics

Promensencen
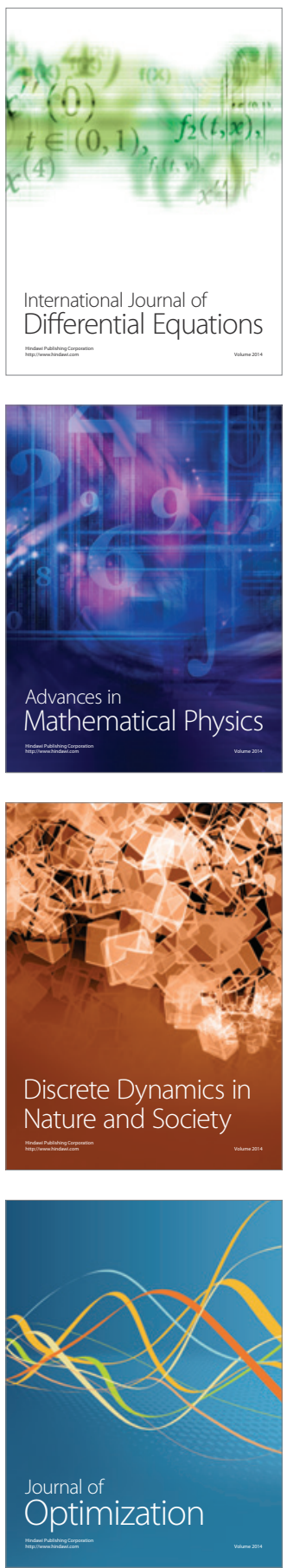